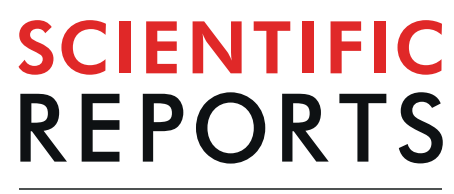

natureresearch

Check for updates

\title{
Predicted effect of ticagrelor on the pharmacokinetics of dabigatran etexilate using physiologically based pharmacokinetic modeling
}

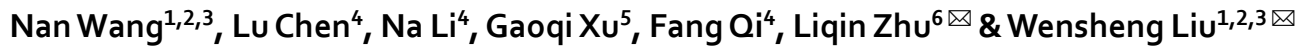

Dabigatran etexilate (DABE) is a direct oral anticoagulant (DOAC) and may be combined with ticagrelor, a $\mathrm{P} 2 \mathrm{Y}_{12}$ inhibitor with antiplatelet effects. This combination of antiplatelet drugs and anticoagulants would increases the risk of bleeding in patients. In addition, the potential drug interaction may further increase the risk of bleeding. At present, there is scarce research to clarify the results of the interaction between the two. Therefore, we conducted this study to identify the potential impact of ticagrelor on the pharmacokinetics of DABE using physiologically based pharmacokinetic (PBPK) modeling. The models reasonably predicted the concentration-time profiles of dabigatran (DAB), the transformation form after DABE absorption, and ticagrelor. For pharmacokinetic drug-drug interaction (DDI), exposure to $D A B$ at steady state was increased when co-administrated with ticagrelor. The $C_{\max }$ and $A U C_{0-t}$ of DAB were raised by approximately $8.7 \%$ and $7.1 \%$, respectively. Meanwhile, a stable-state ticagrelor coadministration at $400 \mathrm{mg}$ once-daily increased the $C_{\max }$ and $A U C_{0-t}$ of DAB by approximately $12.8 \%$ and $18.8 \%$, respectively. As conclusions, Ticagrelor slightly increased the exposure of DAB. It is possible to safely use ticagrelor in a double or triple antithrombotic regimen containing $D A B E$, only considering the antithrombotic efficacy, but not need to pay much attention on the pharmacokinetic DDI.
\end{abstract}

Previously referred to as new oral anticoagulants, the direct oral anticoagulants (DOACs) are a group of rapidly acting and directly clotting factors inhibiting anticoagulants. Current available DOACs include dabigatran etexilate (DABE), rivaroxaban, apixaban and edoxaban ${ }^{1}$. Among the DOACs, DABE is a particular Factor IIa (thrombin) inhibitor, used for (1) the prevention of stroke and systemic embolism in patients with nonvalvular atrial fibrillation $(\mathrm{AF})^{2},(2)$ the treatment of acute venous thromboembolism and prevention of venous thromboembolism recurrence ${ }^{3}$, and (3) the prevention of venous thromboembolism after hip or knee replacement surgery ${ }^{4}$. $\mathrm{DABE}$ is a prodrug, which is converted to the active form dabigatran (DAB) after absorption. Both the prodrug and the metabolite are excreted via the renal route ${ }^{5}$, and not involved in the cytochrome P450 (CYP450) enzymes, regarding as an advantage over warfarin and many other DOACs. However, DABE, but not DAB, is a substrate for permeability glycoprotein (P-gp) ${ }^{2}$, which is an important efflux transporter protein, affecting the absorption of $\mathrm{DABE}^{6}$. Therefore, a P-gp inhibitor, such as amiodarone, verapamil, ketoconazole, clarithomycin, may increase the area under the plasma concentration-time curve (AUC) of DAB from about $50 \%$ to over $200 \%{ }^{7}$. Besides, the P-gp mediated drug interactions between DABE and antibiotics, proton pump inhibitors, antiepileptic drugs or some new antiretroviral/antiproliferative drugs have also been reported ${ }^{8-12}$.

Ticagrelor is a novel, reversible, and direct oral adenosine diphosphate receptor $\mathrm{P}_{2} \mathrm{Y}_{12}$ inhibitor, and is applied in patients with acute coronary syndromes worldwide ${ }^{13-15}$. In the aspect of pharmacokinetics (PK), ticagrelor is both a P-gp substrate and a P-gp inhibitor that may affect substrates transported by the P-gp ${ }^{16}$.

The concomitant use of antagonist and antiplatelet agents can reduce the risk of all-cause death, myocardial infarction (MI), stroke and venous thrombosis in patients suffering from acute coronary syndrome (ACS) ${ }^{17,18}$, and has been extensively applied in patients with both AF and ischemic heart diseases, especially after percutaneous

\footnotetext{
${ }^{1}$ Pharmacy Department, Tianjin Third Central Hospital, Tianjin, China. ${ }^{2}$ Tianjin Key Laboratory of Artificial Cell, Tianjin, China. ${ }^{3}$ Artificial Cell Engineering Technology Research Center of Public Health Ministry, Tianjin, China. ${ }^{4}$ Pharmaceutical College, Tianjin Medical University, Tianjin, China. ${ }^{5}$ Pharmacy Department, Zhejiang Cancer Hospital, Hangzhou, China. ${ }^{6}$ Pharmacy Department, Tianjin First Center Hospital, Tianjin, China. ${ }^{凶}$ e-mail: yzx_ Icyx2@126.com; 1323014021@qq.com
} 
a

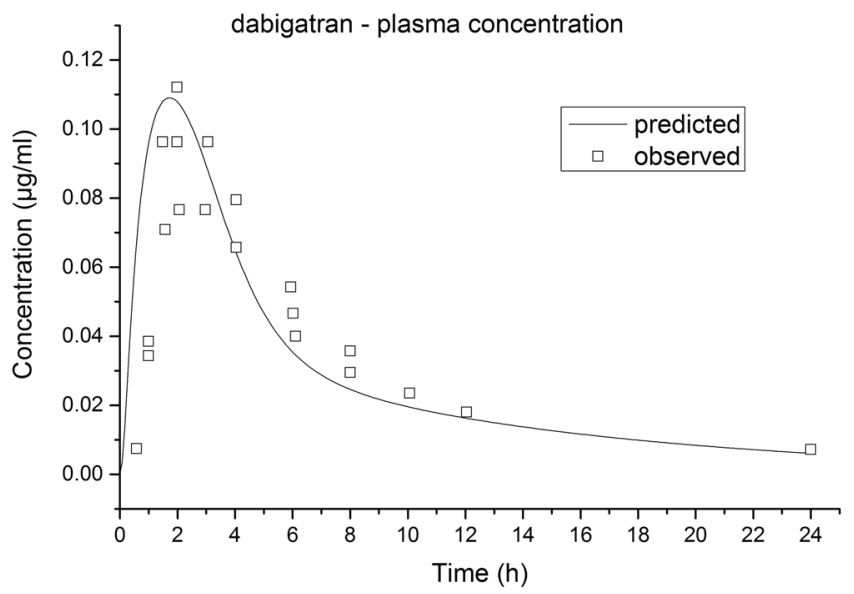

b

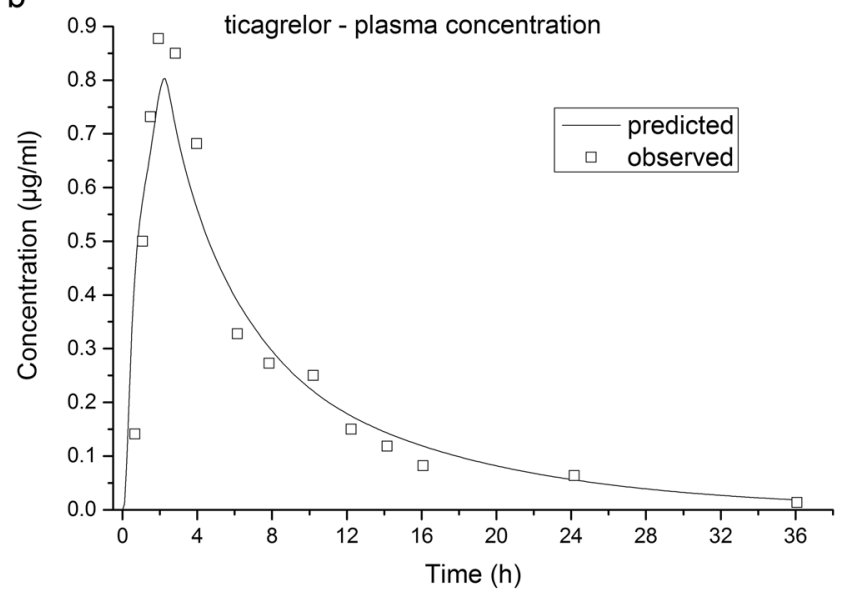

Figure 1. Observed (squares) and physiologically based pharmacokinetic (PBPK) model-stimulated (lines) plasma concentration-time profile of dabigatran and ticagrelor: (a) dabigatran $150 \mathrm{mg}$ oral (b) ticagrelor $200 \mathrm{mg}$ oral.

\begin{tabular}{|c|c|c|c|}
\hline Parameters & Unit & \begin{tabular}{|l|} 
Dabigatran \\
Etexilate
\end{tabular} & Ticagrelor \\
\hline \multicolumn{4}{|c|}{ Physicochemical parameters } \\
\hline Molecular weight & $\mathrm{g} / \mathrm{mol}$ & $627.73^{\mathrm{a}}$ & $522.57^{\mathrm{a}}$ \\
\hline Dosage form & & Capsule & Tablet \\
\hline $\log \mathrm{P}$ & & $4.59^{\mathrm{a}}$ & $2.28^{\mathrm{a}}$ \\
\hline $\mathrm{pKa}$ (acid) & & $17.89^{\mathrm{a}}$ & $12.94^{\mathrm{a}}$ \\
\hline fu (plasma) & $\%$ & $65 \%^{\mathrm{a}}$ & $0.2 \%^{\mathrm{a}}$ \\
\hline Aqueous solubility & $\mathrm{mg} / \mathrm{mL}$ & $1.8^{\mathrm{a}}$ & $0.063^{\mathrm{a}}$ \\
\hline $\mathrm{R}_{\mathrm{bp}}$ & fold & $3.08^{\mathrm{b}}$ & $0.4^{\mathrm{b}}$ \\
\hline $\mathrm{P}_{\text {eff }}$ & $\mathrm{cm} / \mathrm{s} * 10^{4}$ & $1^{\mathrm{b}}$ & $2^{\mathrm{b}}$ \\
\hline \multicolumn{4}{|l|}{ Metabolism data } \\
\hline P-gp $V_{\max }$ & & $10^{c}$ & \\
\hline P-gp K & $\mu \mathrm{M}$ & $1.0^{c}$ & \\
\hline
\end{tabular}

Table 1. Summary of model parameters used in simulations. $\mathrm{pKa}$, acid dissociation constant; $\mathrm{P}_{\text {eff }}$, effective permeability; $\mathrm{R}_{\mathrm{bp}}$, blood/plasma concentration ratio; fu (plasma), fraction unbound in plasma; logP, partition coefficient. ${ }^{a}$ From DrugBank (https://www.drugbank.ca/). ${ }^{b}$ Estimated by ADMET Predictor. ${ }^{~}$ Zhao Y. et al. (2014).

coronary intervention (PCI $)^{19,20}$. As new antithrombotic drugs, DABE in combination with ticagrelor offers an additional option to substitute warfarin regimen, with the expectation of decreasing the bleeding risk and simultaneously maintaining the clinical effect ${ }^{21}$. 


\begin{tabular}{|c|c|c|c|c|c|}
\hline & & $\begin{array}{l}\mathrm{C}_{\max } \\
(\mu \mathrm{g} / \\
\mathrm{mL})\end{array}$ & $\begin{array}{l}\mathrm{T}_{\max } \\
\text { (h) }\end{array}$ & $\begin{array}{l}\mathrm{AUC}_{\text {0-inf }} \\
(\mu \mathrm{g} \cdot \mathrm{h} / \\
\mathrm{mL})\end{array}$ & $\begin{array}{l}\mathrm{AUC}_{0-\mathrm{t}} \\
(\mu \mathrm{g} \cdot \mathrm{h} / \\
\mathrm{mL})\end{array}$ \\
\hline \multirow{3}{*}{ Ticagrelor } & Observed & 0.88 & 1.92 & 6.63 & 6.52 \\
\hline & Predicted & 0.80 & 2.28 & 6.76 & 6.56 \\
\hline & Fold-error & 1.10 & 0.84 & 0.98 & 0.99 \\
\hline \multirow{3}{*}{ Dabigatran } & Observed & 0.11 & \begin{tabular}{|l|}
1.97 \\
\end{tabular} & 0.79 & 0.70 \\
\hline & Predicted & 0.11 & 1.76 & 0.76 & 0.69 \\
\hline & Fold-error & 1.00 & 1.12 & 1.04 & 1.01 \\
\hline
\end{tabular}

Table 2. Observed and simulated pharmacokinetic parameters of ticagrelor and dabigatran etexilate. $\mathrm{C}_{\max }$, maximum plasma concentration; $\mathrm{T}_{\max }$, time from last dosing to the maximum plasma concentration; $\mathrm{AUC}_{0 \text {-inf, }}$ area under the concentration-time curve over the simulated period; $\mathrm{AUC}_{0-\mathrm{t}}$, area under the concentration-time curve till infinite.

\begin{tabular}{|l|l|l|l|l|}
\hline & $\begin{array}{l}\mathbf{C}_{\max } \\
(\mu \mathrm{g} / \\
\mathbf{m L})\end{array}$ & $\begin{array}{l}\mathbf{T}_{\max } \\
(\mathbf{h})\end{array}$ & $\begin{array}{l}\mathbf{A U C}_{\text {-tt }} \\
(\mu \mathrm{g} \cdot \mathbf{h} / \\
\mathbf{m L})\end{array}$ & $\begin{array}{l}\mathbf{A U C}_{\text {0-inf }} \\
(\mu \mathrm{g} \cdot \mathbf{h} / \\
\mathbf{m L})\end{array}$ \\
\hline Ticagrelor-baseline/DDI & 13.48 & 123.4 & 1190000 & 98300000 \\
\hline Dabigatran-baseline & 0.138 & 121.3 & 8334.9 & 8452.9 \\
\hline Dabigatran-DDI & 0.15 & 121.3 & 8925.4 & 9073.3 \\
\hline Dabigatran-ratio & 1.087 & 1 & 1.071 & 1.073 \\
\hline
\end{tabular}

Table 3. Simulated pharmacokinetic parameters of dabigatran following multiple administrations of $150 \mathrm{mg}$ twice-daily dabigatran etexilate alone or with multiple dose of ticagrelor at $90 \mathrm{mg}$ twice daily. $\mathrm{C}_{\max }$, maximum plasma concentration; $\mathrm{T}_{\max }$, time from last dosing to the maximum plasma concentration; $\mathrm{AUC}_{0 \text {-inf }}$, area under the concentration-time curve over the simulated period; $\mathrm{AUC}_{0-\mathrm{t}}$, area under the concentration-time curve till infinite; DDI, drug-drug interaction.

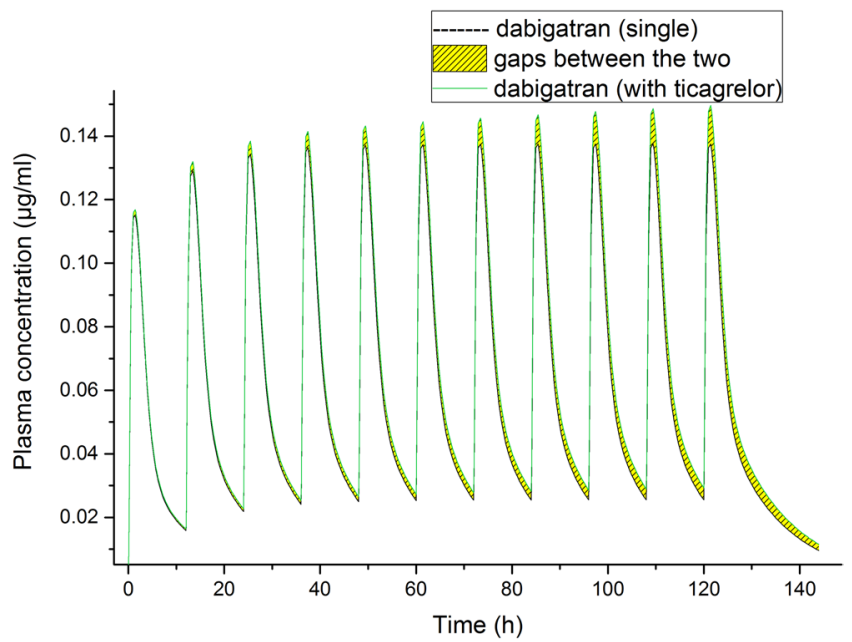

Figure 2. Stimulated mean plasma concentrations of dabigatran over time following multiple administrations of $150 \mathrm{mg}$ twice-daily dabigatran etexilate alone or with multiple dose of ticagrelor at $90 \mathrm{mg}$ twice daily.

Thus, researchers have focused on the antithrombotic activation and effect of preventing cardiovascular events of DABE in conjunction with ticagrelor ${ }^{22,23}$, reaching conclusions that a triple therapy of DABE in combination with ticagrelor plus aspirin is as effective as warfarin triple regimen ${ }^{22}$, and dual therapy of DABE plus a P2 $\mathrm{Y}_{12}$ inhibitor (clopidogrel or ticagrelor) is non-inferior to triple therapy of warfarin plus a $\mathrm{P}_{2} \mathrm{Y}_{12}$ inhibitor (clopidogrel or ticagrelor) and aspirin among patients with atrial fibrillation after PCI, and the risk of bleeding is lower ${ }^{23}$.

However, the potential P-gp induced drug-drug interaction (DDI) between DABE and ticagrelor, which may lead to an elevation of DAB plasma concentration, has not been accurately described. In addition, the indications for this dual therapy are still being explored. Our study aims to use a physiologically based pharmacokinetic (PBPK) model to predict PK profiles and to assess the P-gp mediated DDI of DABE when co-administered with multiple doses of ticagrelor. 


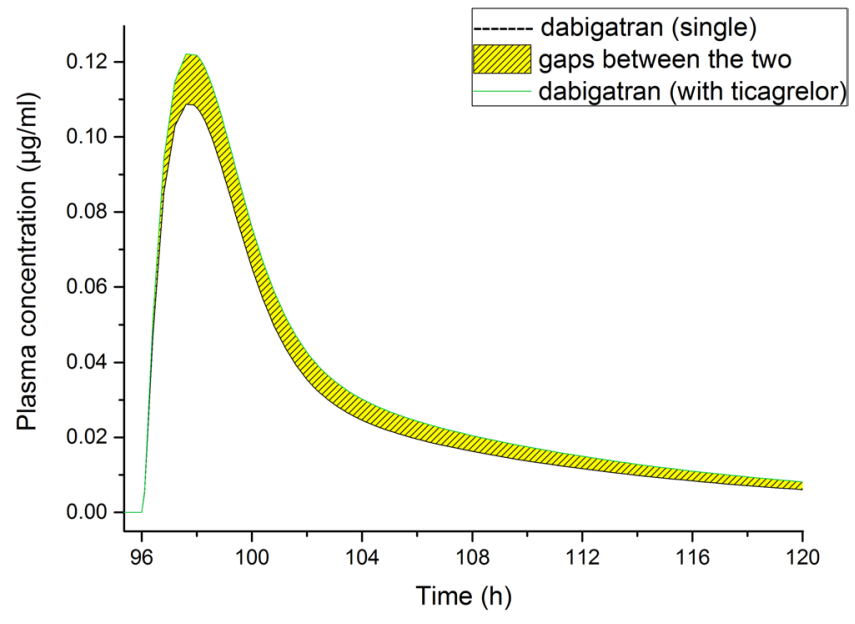

Figure 3. Stimulated mean plasma concentrations of dabigatran over time following multiple administrations of a single $150 \mathrm{mg}$ dose dabigatran etexilate alone or with a fore-4-dose (4 days) ticagrelor at $400 \mathrm{mg}$.

\begin{tabular}{|l|l|l|l|l|}
\hline & $\begin{array}{l}\mathrm{C}_{\max } \\
(\mu \mathrm{g} / \\
\mathbf{m L})\end{array}$ & $\begin{array}{l}\mathrm{T}_{\max } \\
(\mathbf{h})\end{array}$ & $\begin{array}{l}\text { AUC } \\
(\mu \mathrm{g} \cdot \mathbf{h} / \\
\mathbf{m L})\end{array}$ & $\begin{array}{l}\mathbf{A U C}_{\text {0-inf }} \\
(\mu \mathrm{g} \cdot \mathbf{h} / \\
\mathbf{m L})\end{array}$ \\
\hline Ticagrelor-baseline/DDI & 24.2 & 106 & 1700000 & 16100000 \\
\hline Dabigatran-baseline & 0.109 & 97.7 & 693.4 & 768.2 \\
\hline Dabigatran-DDI & 0.123 & 97.8 & 823.7 & 931 \\
\hline Dabigatran-ratio & 1.128 & 1.001 & 1.188 & 1.212 \\
\hline
\end{tabular}

Table 4. Simulated pharmacokinetic parameters of dabigatran following multiple administrations of a single $150 \mathrm{mg}$ dose dabigatran etexilate alone or with a fore-4-dose (4 days) ticagrelor at $400 \mathrm{mg}$. $\mathrm{C}_{\max }$, maximum plasma concentration; $\mathrm{T}_{\max }$, time from last dosing to the maximum plasma concentration; $\mathrm{AUC}_{0 \text {-inf }}$, area under the concentration-time curve over the simulated period; $\mathrm{AUC}_{0-\mathrm{t}}$, area under the concentration-time curve till infinite; DDI, drug-drug interaction.

\section{Results}

Physiologically based pharmacokinetic model and verification. We simulated blood concentration models of $150 \mathrm{mg}$ dabigatran and $200 \mathrm{mg}$ ticagrelor. Blood concentration data at each time point see Supplementary Table 1 and Supplementary Table 2.

The models reasonably predicted the concentration-time profiles of $\mathrm{DAB}\left(\mathrm{C}_{\max } 0.11 \mu \mathrm{g} / \mathrm{mL}\right.$ predicted vs. $0.11 \mu \mathrm{g} / \mathrm{mL}$ observed, $\mathrm{T}_{\max } 1.76 \mathrm{~h}$ predicted vs. $1.97 \mathrm{~h}$ observed, AUC0-t $0.69 \mu \mathrm{g} \cdot \mathrm{h} / \mathrm{mL}$ predicted vs. $0.70 \mu \mathrm{g} \cdot \mathrm{h} /$ $\mathrm{mL}$ observed) and ticagrelor $\left(\mathrm{C}_{\max } 0.80 \mu \mathrm{g} / \mathrm{mL}\right.$ predicted vs. $0.88 \mu \mathrm{g} / \mathrm{mL}$ observed, $\mathrm{T}_{\max } 2.28 \mathrm{~h}$ predicted vs. $1.92 \mathrm{~h}$ observed, AUC0-t $6.56 \mu \mathrm{g} \cdot \mathrm{h} / \mathrm{mL}$ predicted vs. $6.52 \mu \mathrm{g} \cdot \mathrm{h} / \mathrm{mL}$ observed).

The in vivo data were loaded to verify the predictive accuracy. The simulations and verifications of the plasma concentration-time curves for DAB at a dose of DABE $150 \mathrm{mg}$ and ticagrelor at a dose of $200 \mathrm{mg}$ were shown in Fig. 1a,b, respectively, revealing that the simulated profiles for both $\mathrm{DAB}$ and ticagrelor were qualitatively similar to the observed data.

The simulated plasma concentration-time profiles of DAB and ticagrelor corresponded well with the observed data obtained from literatures ${ }^{24,25}$. In addition, the predicted PK parameters were reasonably consistent $(<2$-fold error) with the observed values, which indicated that the models were successfully and accurately simulated the pharmacokinetic process of the two medications. The predicted and observed PK parameters with prediction accuracy were summarized in Table 2.

Drug-drug Interaction simulation with DABE and ticagrelor. A dynamic DDI simulation was performed to predict the effect of ticagrelor on the PK of DABE, using multiple doses in the PBPK model for 5 days (10 doses). The plasma concentration-time curves of DAB at baseline and following DDI were shown in Fig. 2, blood concentration data in Supplementary Table 3. The model-predicted ratios of DAB $C_{\max }$ and $\mathrm{AUC}_{0-\mathrm{t}}$ with ticagrelor co-administration were 1.087 and 1.071 (Table 3).

In another DDI simulation, DABE were started after ticagrelor reaching stable state. The plasma concentration-time curves of DAB at baseline and following DDI after ticagrelor reaching stable state were drawn in Fig. 3, blood concentration data in Supplementary Table 4. The ratios of $\mathrm{C}_{\max }$ and $\mathrm{AUC}_{0-\mathrm{t}}$ values were ca. 1.128 and 1.188 , respectively (Table 4). The result showed that the predicted ratio of $\mathrm{DAB} A \mathrm{AC}_{0-\mathrm{t}}$ was higher than the ratios observed in the former regimen. 


\section{Discussion}

$\mathrm{DABE}$ is a new oral anticoagulant used to reduce the risk of stroke and systemic embolism in non-valvular atrial fibrillation and to treat and prevent blood clots in veins. In the RE-DEEM trial ${ }^{26}$, DABE was given in addition to dual antiplatelet treatment (aspirin plus clopidogrel) in patients with a recent myocardial infarction at high risk of new ischaemic cardiovascular events. Ticagrelor is deemed as an alternative to clopidogrel in patients with acute coronary syndromes, especially with clopidogrel resistance ${ }^{27}$. Currently, concurrent medication with ticagrelor and different anti-coagulation agents has been paid great attention ${ }^{22,23,28-31}$, including DABE plus ticagrelor, which was not yet recommended a few years ago ${ }^{32}$. As mentioned, DABE is a prodrug that has a low oral availability of around $7 \%$, for DABE is an intestinal P-gp substrate ${ }^{6}$. Whereas, in vitro studies have indicated that ticagrelor is a substrate and inhibitor of $\mathrm{P}-\mathrm{gp}^{16}$. The current study was conducted to predict the potential interaction between $\mathrm{DABE}$ and ticagrelor, by comparing the pharmacokinetics of $\mathrm{DAB}$ alone and in combination with ticagrelor using PBPK modeling.

PBPK models are proved useful to integrate all the parameters which affect the pharmacokinetics, for example, the parameters associated with the properties of the drugs and the physiological parameters specific to the animal species. There are now many softwares available (such as GastroPlus, PKsim, Simcyp) which include the parameters, and equations describing the mechanisms involved in drug disposition, metabolism and excretion $^{33}$. PBPK modeling and simulation were performed using the Simcyp Simulator in assessing potential DDIs between DABE and a P-gp inhibitor in renal impairment populations in Doki's study ${ }^{34}$. GastroPlus was used to performed the PBPK models in the prediction of ticagrelor and its active metabolite in liver cirrhosis populations in Zhang's study ${ }^{35}$. Both PBPK models in Zhang's and our study were performed and simulated using GastroPlus $^{\text {TM }}$. Although it is slightly different from Zhang's modeling method, we validated the reliability of the model using data from clinical literature, which prompts the results are credible.

We designed two DDI simulations. One simulation was DABE $150 \mathrm{mg}$ bid Day $1-5+$ ticagrelor (180 mg loading dose followed by $90 \mathrm{mg}$ bid) Day 1-5. Both doses were the maximum common doses currently used in clinical. As a result, a slight increase in $\mathrm{DAB}$ exposure at steady state was observed when co-administrated with ticagrelor - the $\mathrm{C}_{\max }$ and $\mathrm{AUC}_{0-\mathrm{t}}$ of DAB were raised by approximately $8.7 \%$ and $7.1 \%$, respectively. As the DDI was small, we only validated $150 \mathrm{mg}$ DABE dose, and did not put lower dosage into DDI simulation. In the other simulation, we designed a DABE $150 \mathrm{mg}$ single dose after 5 days continuous use of ticagrelor $400 \mathrm{mg}$ qd, which was higher than the approved $90 \mathrm{mg}$ bid maintenance dose used clinically. This design was based on the previous study of the interaction between ticagrelor and digoxin ${ }^{36}$. In this dose regimen, ticagrelor increased the $\mathrm{C}_{\max }$ and $\mathrm{AUC}_{0-\mathrm{t}}$ of DAB by approximately $12.8 \%$ and $18.8 \%$, respectively, indicating that there was limited influence of ticagrelor on the PK parameters of DAB, even at a relatively high ticagrelor level.

In a previous study, Weisshaar et al investigated the pharmacodynamic and pharmacokinetic effect of orally administered ticagrelor and aspirin in combination with DABE in healthy male subjects, compared with DABE alone $^{22}$. As a result, the median DAB plasma concentration was increased $130 \%$ at $3 \mathrm{~h}$ after concomitant use of $\mathrm{DABE}$, ticagrelor and aspirin, versus single-dose DABE alone. Besides, in the summary of product characteristics of DABE on European Medicines Agency (EMA), drug interaction data for DABE-ticagrelor showed a widely varied $A U C$ and $C_{\max }$ increase, which were higher than the change in our model prediction. We analyzed the possible reasons were as follows: First, the research population was different. The research population of our study was healthy young people with normal body weight, while the results listed in the EMA Summary of product characteristics may be the result of a combination of various population. Second, from a mechanistic perspective, ticagrelor is a weak P-gp inhibitor, which has a certain effect on drug transport, but the inhibitory effect is relatively weak. Compared with placebo, the AUC $\tau$ of digoxin, a substrate of renal and intestinal P-gp, was increased by approximately and $28 \%$ when co-administration with ticagrelor ${ }^{36}$. Similarly, ticagrelor increased the $\mathrm{C}_{\max }$ and $\mathrm{AUC}_{0 \text {-inf }}$ of cyclosporine, another substrate of intestinal P-gp ${ }^{37}$, by approximately $5 \%$ and $12 \%$, respectively ${ }^{38}$, proving ticagrelor is a weak inhibitor of intestinal P-gp. Therefore, our results were reasonable from the perspective of mechanism analysis.

With respect to efficacy, in patients with atrial fibrillation who had undergone PCI, the risk of bleeding was lower and non-inferior among those who receive dual therapy with DABE and ticagrelor (or clopidogrel), comparing to those who receive triple therapy with warfarin, ticagrelor (or clopidogrel) and aspirin, in RE-DUAL trail ${ }^{23}$. Regrettably, ticagrelor was not grouped separately, so that we were wondering whether the satisfactory efficacy was corresponding with the DDI between DABE and ticagrelor. Besides, further studies are in need of conducting to explore this dual therapy in the other indications for combination.

From another aspect, since both CYP3A4 and P-gp were involved in the disposition of rivaroxaban, apixaban and edoxaban ${ }^{39,40}$, clinical consequences may be affected when one of the three xabans was co-administrated with ticagrelor. In GEMINI-ACS-1 trial ${ }^{41}$, the frequency of TIMI non-CABG relevant bleeding for rivaroxaban plus ticagrelor was significantly increased compared to rivaroxaban plus clopidogrel. This result may partially blame on the DDI between rivaroxaban and ticagrelor. Therefore, it may be more secure to combine ticagrelor with DABE than other DOACs when using the routine dosage, for the pharmacokinetic changes of DABE are limited. Besides, it is possible to be a safety choice to use ticagrelor in a double or triple antithrombotic therapy with DABE.

Although the increases of DAB plasma concentration were considered unlikely to be of clinical significance, close clinical and laboratory monitoring was still highly recommended, referring to the higher rate of total bleeding at $33.3 \% / 27.1 \%$ in patients receiving DABE $150 \mathrm{mg} / 110 \mathrm{mg}$ plus ticagrelor (or clopidogrel) ${ }^{23}$, compared with the rate at $16.42 \% / 14.62 \%$ with DABE $150 \mathrm{mg} / 110 \mathrm{mg}$ alone ${ }^{42}$ or $16.1 \%$ with ticagrelor alone ${ }^{13}$, particularly in special populations such as elderly, renal dysfunction and other patients with high risk of bleeding. As DAB is mainly excreted by the kidneys, concomitant renal insufficiency may further increase DAB exposure when given together with P-gp inhibitors. This theoretically supports the findings of the literature, which found impaired renal function, co-medication with antiplatelet drugs or P-gp inhibitors are the risk factors for bleeding with DOACs ${ }^{43}$. 
Overall, the results of this study fill in gaps in the effect of ticagrelor on the PK parameters of DAB, so as to provide support for the clinical application and further research of the combination of these two drugs.

There are several limitations to the current work. First, virtual Caucasian healthy male adults were simulated in the study, lacking for the evaluation on the other population like the elderly, female etc. A human study revealed that $\mathrm{DAB}$ exposure in female Caucasian healthy volunteers was about $25 \%$ higher than in males. This gender difference is most likely to be attributable to the lower body weight and lower creatinine clearance in females, which in turn results in lower drug clearance than males ${ }^{44}$. Second, the inhibitory effects of ticagrelor on other DOACs, i.e. rivaroxaban, apixaban, edoxaban, were not assessed, due to the limitation of published in vitro P-gp transporter study specifically designed to capture certain parameters that is required in the process of DDI predicting. Finally, the efficacy and safety of this co-administration therapy should be further verified by more clinical experience and experiments.

\section{Conclusions}

DABE and ticagrelor showed a pharmacokinetic interaction. At steady-state, DAB $\mathrm{C}_{\max }$ and $\mathrm{AUC}_{0-\mathrm{t}}$ were increased by $8.5 \%$ and $7.1 \%$, respectively, in the presence of ticagrelor versus DABE alone. Meanwhile, after a 4-day loading of ticagrelor, $\mathrm{DAB} \mathrm{C}_{\max }$ and $\mathrm{AUC}_{0-\mathrm{t}}$ were increased by $12.8 \%$ and $18.8 \%$, respectively, versus $\mathrm{DABE}$ alone. Since the changes in pharmacokinetic parameters are limited, it is possible to safely use ticagrelor in a double or triple antithrombotic therapy with DABE. Based on these findings, it is recommended that DABE and ticagrelor can be used concomitantly, only considering the antithrombotic efficacy, but not need to pay much attention on the pharmacokinetic DDI.

\section{Methods}

The construction of PBPK models and the simulation of drug interaction were performed with GastroPlus v 9.0 (Simulations Plus Inc. Lancaster, CA). The models were constructed and refined to match the observed maximum plasma concentration $\left(\mathrm{C}_{\max }\right)$ and area under the plasma concentration-time curve (AUC) values from reported literatures. The Population Estimates for Age-Related (PEAR) human physiology model was used to assume that the typical study subjects were 30 -year-old American healthy males weighing $78 \mathrm{~kg}$.

Structure and validation of DAB and ticagrelor PBPK models. The PBPK models were developed via known physicochemical and $\mathrm{PK}$ parameters, which were initially required to run simulations with GastroPlus, including: formulation; molecular weight; partition coefficient $(\log \mathrm{P})$; the acid dissociation constant $(\mathrm{pKa})$; fraction unbound in plasma $\left(\mathrm{f}_{\mathrm{up}}\right)$; aqueous solubility; blood/plasma concentration ratio $\left(\mathrm{R}_{\mathrm{bp}}\right)$; effective permeability $\left(\mathrm{P}_{\text {eff }}\right)$. These parameters were obtained from DrugBank (https://www.drugbank.ca/) or acquired from the ADMET Predictor (Simulations Plus Inc.) which is an inbuilt module within GastroPlus. Adjusted plasma $f_{u p}$ values were used in the models. The P-gp and kinetic inputs such as maximum reaction velocity $\left(\mathrm{V}_{\max }\right)$ and Michaelis-Menten constant $\left(\mathrm{K}_{\mathrm{m}}\right)$ were obtained from the published literature ${ }^{24}$. All the parameters were listed in Table 1.

After the base PBPK models were constructed, simulations were conducted with an initial dose of DABE $150 \mathrm{mg}$ or ticagrelor $200 \mathrm{mg}$. The predicted plasma concentration-time curves were validated using data from experimental human studies where volunteers received a single dose of DABE $150 \mathrm{mg}$ or ticagrelor $200 \mathrm{mg}$. The overall accuracy of the predicted PK parameters was assessed by the fold-error (difference between predicted and observed in vivo values), and the prediction was considered successful if the fold-error was $<2^{45}$.

Quantitative prediction of drug-drug interaction. The DDI between DABE and ticagrelor in virtual healthy volunteers were simulated in the PBPK model, using GastroPlus, to predict the inhibitory effect of ticagrelor on the plasma concentration-time data of DAB.

The in vitro inhibition constant $\left(\mathrm{K}_{\mathrm{i}}\right)$ of ticagrelor on human P-gp was calculated from Eq1 with published data $^{24,36}$.

$$
\mathrm{ki}=\frac{1}{0} \cdot 4=1 \cdot \frac{5}{0} \cdot 4=3 \cdot 75 \mu \mathrm{m}
$$

Dynamic simulations of DAB plasma concentration-time profiles taken with and without ticagrelor were run with the DDI Module within GastroPlus. The dose and dose interval of the substrate and inhibitor were set based on the US Food and Drug Administration (FDA) drug instructions.

All virtual subjects received oral DABE $150 \mathrm{mg}$ twice-daily (Day 1-5). Besides, a loading dose of $180 \mathrm{mg}$ and maintenance doses of $90 \mathrm{mg}$ twice-daily ticagrelor was co-administered with DABE on Day 1-5, to determine the inhibitory activity.

The DDI was further evaluated in another PBPK model, in which, ticagrelor at $400 \mathrm{mg}$ once daily ${ }^{36}$ was delivered on days 1-4, and then, DABE at $150 \mathrm{mg}$ was added to the 5th dose of ticagrelor, when ticagrelor had reached stable state.

A number of PK parameters of DABE were predicted by the DDI models. These parameters included $\mathrm{C}_{\max }$, time from last dosing to the maximum concentration of the analyte in plasma at steady state $\left(\mathrm{T}_{\max }\right)$ and area under the concentration-time curve at steady state over the simulated period $\left(\mathrm{AUC}_{0-\mathrm{t}}\right)$ and till infinite $\left(\mathrm{AUC}_{0 \text {-inf }}\right)$. In addition, DAB concentration-time curves for both models were depicted.

Received: 27 December 2019; Accepted: 22 May 2020;

Published online: 16 June 2020 


\section{References}

1. Gibson, C. M. \& Finks, S. W. Edoxaban: How Does the Newest Agent Fit into the DOAC Landscape? The American journal of medicine 130, 900-906, https://doi.org/10.1016/j.amjmed.2017.02.048 (2017).

2. Blair, H. A. \& Keating, G. M. Dabigatran Etexilate: A Review in Nonvalvular Atrial Fibrillation. Drugs 77, 331-344, https://doi. org/10.1007/s40265-017-0699-z (2017).

3. Greig, S. L. \& McKeage, K. Dabigatran etexilate: a review of its use in the treatment of acute venous thromboembolism and prevention of venous thromboembolism recurrence. Drugs 74, 1785-1800, https://doi.org/10.1007/s40265-014-0304-7 (2014).

4. Burness, C. B. \& McKeage, K. Dabigatran etexilate: a review of its use for the prevention of venous thromboembolism after total hip or knee replacement surgery. Drugs 72, 963-986, https://doi.org/10.2165/11209080-000000000-00000 (2012).

5. Stangier, J. Clinical pharmacokinetics and pharmacodynamics of the oral direct thrombin inhibitor dabigatran etexilate. Clinical pharmacokinetics 47, 285-295, https://doi.org/10.2165/00003088-200847050-00001 (2008).

6. Chin, P. K., Vella-Brincat, J. W., Barclay, M. L. \& Begg, E. J. Perspective on dabigatran etexilate dosing: why not follow standard pharmacological principles? British journal of clinical pharmacology 74, 734-740, https://doi.org/10.1111/j.1365-2125.2012.04266.x (2012).

7. Delavenne, X. et al. A semi-mechanistic absorption model to evaluate drug-drug interaction with dabigatran: application with clarithromycin. British journal of clinical pharmacology 76, 107-113, https://doi.org/10.1111/bcp.12055 (2013).

8. Lippi, G., Favaloro, E. J. \& Mattiuzzi, C. Combined administration of antibiotics and direct oral anticoagulants: a renewed indication for laboratory monitoring? Seminars in thrombosis and hemostasis 40, 756-765, https://doi.org/10.1055/s-0034-1381233 (2014).

9. Ollier, E. et al. In vitro and in vivo evaluation of drug-drug interaction between dabigatran and proton pump inhibitors. Fundamental \& clinical pharmacology 29, 604-614, https://doi.org/10.1111/fcp.12154 (2015).

10. Stollberger, C. \& Finsterer, J. Interactions between non-vitamin K oral anticoagulants and antiepileptic drugs. Epilepsy research 126, 98-101, https://doi.org/10.1016/j.eplepsyres.2016.06.003 (2016).

11. Kumar, P. et al. Differential Influence of the Antiretroviral Pharmacokinetic Enhancers Ritonavir and Cobicistat on Intestinal P-Glycoprotein Transport and the Pharmacokinetic/Pharmacodynamic Disposition of Dabigatran. Antimicrobial agents and chemotherapy 61, https://doi.org/10.1128/AAC.01201-17 (2017).

12. Hsyu, P. H., Pignataro, D. S. \& Matschke, K. Effect of bosutinib on the absorption of dabigatran etexilate mesylate, a P-glycoprotein substrate, in healthy subjects. European journal of clinical pharmacology 73, 57-63, https://doi.org/10.1007/s00228-016-2115-0 (2017).

13. Wallentin, L. et al. Ticagrelor versus clopidogrel in patients with acute coronary syndromes. The New England journal of medicine 361, 1045-1057, https://doi.org/10.1056/NEJMoa0904327 (2009).

14. James, S. et al. Ticagrelor versus clopidogrel in acute coronary syndromes in relation to renal function: results from the Platelet Inhibition and Patient Outcomes (PLATO) trial. Circulation 122, 1056-1067, https://doi.org/10.1161/ CIRCULATIONAHA.109.933796 (2010).

15. Dhillon, S. Ticagrelor: a review of its use in adults with acute coronary syndromes. American journal of cardiovascular drugs : drugs, devices, and other interventions 15, 51-68, https://doi.org/10.1007/s40256-015-0108-5 (2015).

16. Teng, R. Ticagrelor: Pharmacokinetic, Pharmacodynamic and Pharmacogenetic Profile: An Update. Clinical pharmacokinetics 54, 1125-1138, https://doi.org/10.1007/s40262-015-0290-2 (2015).

17. Andreotti, F., Testa, L., Biondi-Zoccai, G. G. \& Crea, F. Aspirin plus warfarin compared to aspirin alone after acute coronary syndromes: an updated and comprehensive meta-analysis of 25,307 patients. European heart journal 27, 519-526, https://doi. org/10.1093/eurheartj/ehi485 (2006).

18. Rothberg, M. B., Celestin, C., Fiore, L. D., Lawler, E. \& Cook, J. R. Warfarin plus aspirin after myocardial infarction or the acute coronary syndrome: meta-analysis with estimates of risk and benefit. Annals of internal medicine 143, 241-250 (2005).

19. Cairns, J. A. \& McMurtry, M. S. Oral antithrombotic therapy in atrial fibrillation associated with acute or chronic coronary artery disease. The Canadian journal of cardiology 29, S60-70, https://doi.org/10.1016/j.cjca.2013.04.006 (2013).

20. Dezsi, C. A., Dezsi, B. B. \& Dezsi, D. A. Antithrombotic treatment in anticoagulated atrial fibrillation patients undergoing percutaneous coronary intervention. European journal of internal medicine 40, 1-7, https://doi.org/10.1016/j.ejim.2017.01.001 (2017).

21. Cannon, C. P. et al. Design and Rationale of the RE-DUAL PCI Trial: A Prospective, Randomized, Phase $3 \mathrm{~b}$ Study Comparing the Safety and Efficacy of Dual Antithrombotic Therapy With Dabigatran Etexilate Versus Warfarin Triple Therapy in Patients With Nonvalvular Atrial Fibrillation Who Have Undergone Percutaneous Coronary Intervention With Stenting. Clinical cardiology 39, 555-564, https://doi.org/10.1002/clc.22572 (2016).

22. Weisshaar, S. et al. Antithrombotic triple therapy and coagulation activation at the site of thrombus formation: a randomized trial in healthy subjects. Journal of thrombosis and haemostasis : JTH 12, 1850-1860, https://doi.org/10.1111/jth.12726 (2014).

23. Cannon, C. P. et al. Dual Antithrombotic Therapy with Dabigatran after PCI in Atrial Fibrillation. The New England journal of medicine 377, 1513-1524, https://doi.org/10.1056/NEJMoa1708454 (2017).

24. Zhao, Y. \& Hu, Z. Y. Physiologically based pharmacokinetic modelling and in vivo [I]/K(i) accurately predict P-glycoproteinmediated drug-drug interactions with dabigatran etexilate. British journal of pharmacology 171, 1043-1053, https://doi.org/10.1111/ bph.12533 (2014)

25. Teng, R., Oliver, S., Hayes, M. A. \& Butler, K. Absorption, distribution, metabolism, and excretion of ticagrelor in healthy subjects. Drug metabolism and disposition: the biological fate of chemicals 38, 1514-1521, https://doi.org/10.1124/dmd.110.032250 (2010).

26. Oldgren, J. et al. Dabigatran vs. placebo in patients with acute coronary syndromes on dual antiplatelet therapy: a randomized, double-blind, phase II trial. European heart journal 32, 2781-2789, https://doi.org/10.1093/eurheartj/ehr113 (2011).

27. Samos, M. et al. Ticagrelor: a safe and effective approach for overcoming clopidogrel resistance in patients with stent thrombosis? Blood coagulation \& fibrinolysis : an international journal in haemostasis and thrombosis 27, 117-120, https://doi.org/10.1097/ MBC.0000000000000406 (2016).

28. Braun, O. O. et al. Concomitant use of warfarin and ticagrelor as an alternative to triple antithrombotic therapy after an acute coronary syndrome. Thrombosis research 135, 26-30, https://doi.org/10.1016/j.thromres.2014.10.016 (2015).

29. Lu, W. et al. Rationale and design of MANJUSRI trial: a randomized, open-label, active-controlled multicenter study to evaluate the safety of combined therapy with ticagrelor and warfarin in AF subjects after PCI-eS. Contemporary clinical trials 40, 166-171, https://doi.org/10.1016/j.cct.2014.12.002 (2015).

30. Gao, F. et al. Rationale and design of the RT-AF study: Combination of rivaroxaban and ticagrelor in patients with atrial fibrillation and coronary artery disease undergoing percutaneous coronary intervention. Contemporary clinical trials 43, 129-132, https://doi. org/10.1016/j.cct.2015.05.012 (2015).

31. Povsic, T. J. et al. A randomized trial to compare the safety of rivaroxaban vs aspirin in addition to either clopidogrel or ticagrelor in acute coronary syndrome: The design of the GEMINI-ACS-1 phase II study. American heart journal 174, 120-128, https://doi. org/10.1016/j.ahj.2016.01.004 (2016).

32. Di Minno, G., Russolillo, A., Gambacorta, C., Di Minno, A. \& Prisco, D. Improving the use of direct oral anticoagulants in atrial fibrillation. European journal of internal medicine 24, 288-294, https://doi.org/10.1016/j.ejim.2013.01.021 (2013).

33. Gobeau, N., Stringer, R., De Buck, S., Tuntland, T. \& Faller, B. Evaluation of the GastroPlus ${ }^{\mathrm{TM}}$ Advanced Compartmental and Transit (ACAT) Model in Early Discovery. Pharmaceutical research 33, 2126-2139, https://doi.org/10.1007/s11095-016-1951-z (2016). 
34. Doki, K., Neuhoff, S., Rostami-Hodjegan, A. \& Homma, M. Assessing Potential Drug-Drug Interactions Between Dabigatran Etexilate and a P-Glycoprotein Inhibitor in Renal Impairment Populations Using Physiologically Based Pharmacokinetic Modeling. CPT: pharmacometrics \& systems pharmacology 8, 118-126, https://doi.org/10.1002/psp4.12382 (2019).

35. Zhang, M. et al. Prediction of Ticagrelor and its Active Metabolite in Liver Cirrhosis Populations Using a Physiologically Based Pharmacokinetic Model Involving Pharmacodynamics. Journal of pharmaceutical sciences 108, 2781-2790, https://doi.org/10.1016/j. xphs.2019.03.028 (2019).

36. Teng, R. \& Butler, K. A pharmacokinetic interaction study of ticagrelor and digoxin in healthy volunteers. European journal of clinical pharmacology 69, 1801-1808, https://doi.org/10.1007/s00228-013-1543-3 (2013).

37. Lown, K. S. et al. Role of intestinal P-glycoprotein (mdr1) in interpatient variation in the oral bioavailability of cyclosporine. Clinical pharmacology and therapeutics 62, 248-260, https://doi.org/10.1016/S0009-9236(97)90027-8 (1997).

38. Teng, R., Kujacic, M. \& Hsia, J. Pharmacokinetic interaction study of ticagrelor and cyclosporine in healthy volunteers. Clinical drug investigation 34, 529-536, https://doi.org/10.1007/s40261-014-0205-2 (2014).

39. Stollberger, C. Drug interactions with new oral anticoagulants in elderly patients. Expert review of clinical pharmacology 10, 1191-1202, https://doi.org/10.1080/17512433.2017.1370369 (2017).

40. Hodin, S. et al. In Vitro Comparison of the Role of P-Glycoprotein and Breast Cancer Resistance Protein on Direct Oral Anticoagulants Disposition. European journal of drug metabolism and pharmacokinetics 43, 183-191, https://doi.org/10.1007/ s13318-017-0434-x (2018).

41. Ohman, E. M. et al. Clinically significant bleeding with low-dose rivaroxaban versus aspirin, in addition to P2Y12 inhibition, in acute coronary syndromes (GEMINI-ACS-1): a double-blind, multicentre, randomised trial. Lancet 389, 1799-1808, https://doi. org/10.1016/S0140-6736(17)30751-1 (2017).

42. Connolly, S. J. et al. Dabigatran versus warfarin in patients with atrial fibrillation. The New England journal of medicine 361, 1139-1151, https://doi.org/10.1056/NEJMoa0905561 (2009).

43. Pfeilschifter, W., Luger, S., Brunkhorst, R., Lindhoff-Last, E. \& Foerch, C. The gap between trial data and clinical practice-an analysis of case reports on bleeding complications occurring under dabigatran and rivaroxaban anticoagulation. Cerebrovascular diseases 36, 115-119, https://doi.org/10.1159/000352062 (2013).

44. Hartter, S., Yamamura, N., Stangier, J., Reilly, P. A. \& Clemens, A. Pharmacokinetics and pharmacodynamics in Japanese and Caucasian subjects after oral administration of dabigatran etexilate. Thrombosis and haemostasis 107, 260-269, https://doi. org/10.1160/TH11-08-0551 (2012).

45. Jones, H. M., Parrott, N., Jorga, K. \& Lave, T. A novel strategy for physiologically based predictions of human pharmacokinetics. Clinical pharmacokinetics 45, 511-542, https://doi.org/10.2165/00003088-200645050-00006 (2006).

\section{Author contributions}

Nan Wang. The main contributor to the research, accomplishing research design, article writing, charting and graphing. Lu Chen. Editing the article, providing support on PBPK modeling and interpretation of some literatures. Na Li. Structuring and validating PBPK models, predicting drug-drug interaction. Gaoqi Xu. Participating in research design. Fang Qi. Help with article writing. Liqin Zhu. Responsible for ideas of the research, providing research funding and platforms, supervising and guiding the entire process of the experiment and the publication of the article, responsible for the entire study. Wensheng Liu. Responsible for ideas of the research, supervising the entire process of the experiment and the publication of the article, responsible for the entire study.

\section{Competing interests}

The authors declare no competing interests.

\section{Additional information}

Supplementary information is available for this paper at https://doi.org/10.1038/s41598-020-66557-X.

Correspondence and requests for materials should be addressed to L.Z. or W.L.

Reprints and permissions information is available at www.nature.com/reprints.

Publisher's note Springer Nature remains neutral with regard to jurisdictional claims in published maps and institutional affiliations.

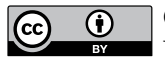

Open Access This article is licensed under a Creative Commons Attribution 4.0 International License, which permits use, sharing, adaptation, distribution and reproduction in any medium or format, as long as you give appropriate credit to the original author(s) and the source, provide a link to the Creative Commons license, and indicate if changes were made. The images or other third party material in this article are included in the article's Creative Commons license, unless indicated otherwise in a credit line to the material. If material is not included in the article's Creative Commons license and your intended use is not permitted by statutory regulation or exceeds the permitted use, you will need to obtain permission directly from the copyright holder. To view a copy of this license, visit http://creativecommons.org/licenses/by/4.0/.

(C) The Author(s) 2020 